\title{
Markers of the Endothelial Glycocalyx Are Improved following Kidney Transplantation
}

\author{
Hui Liew ${ }^{a, b}$ Matthew A. Roberts ${ }^{a, b}$ Lawrence P. McMahon ${ }^{a, b}$ \\ ${ }^{a}$ Department of Renal Medicine, Eastern Health, Box Hill, VIC, Australia; ${ }^{\text {b}}$ Eastern Health Clinical School, Monash \\ University, Melbourne, VIC, Australia
}

\section{Keywords}

Endothelium · Glycocalyx · Transplant · Endothelial dysfunction · Uraemia

\begin{abstract}
Introduction: The endothelial glycocalyx on the vascular luminal surface contributes to endothelial health and function. Damage to this layer is indicative of vascular injury, reflected by increased levels of its shed constituents in serum and an increase in the perfused boundary region (PBR) when measured in sublingual capillaries using the GlycoCheck ${ }^{\mathrm{TM}}$ device. We aimed to examine the longitudinal effects of kidney transplantation on the glycocalyx by measuring biochemical markers of the glycocalyx and endothelial dysfunction and the PBR. Methods: We recruited healthy controls and stage 5 CKD patients scheduled to undergo a kidney transplant. Investigations were performed before transplant and then 1 and 3 months after transplantation. At each point, blood was collected for hyaluronan, syndecan-1, vascular cell adhesion molecule (VCAM-1), and von Willebrand factor (vWF), and a PBR measurement was performed. Results: Thirty healthy controls and 17 patients undergoing a kidney transplant were recruited ( 9 cadaveric and 8 live donation; 12 on dialysis and 5 pre-emptive). Before transplant, transplant recipients had greater evidence of glycocalyx damage than controls. After transplant, PBR improved from median
\end{abstract}

karger@karger.com www.karger.com/kbr

Karger $\stackrel{\text { ' }}{5}$

GOPEN ACCESS
(C) 2021 The Author(s)

Published by S. Karger AG, Basel

This is an Open Access article licensed under the Creative Commons Attribution-NonCommercial-4.0 International License (CC BY-NC) (http://www.karger.com/Services/OpenAccessLicense), applicable to the online version of the article only. Usage and distribution for commercial purposes requires written permission.
2.22 (range 1.29-2.73) to $1.98(1.65-2.25) \mu \mathrm{m}, p=0.024$, and syndecan-1 levels decreased from $98(40-529)$ to $36(20-$ 328) $\mathrm{ng} / \mathrm{mL}, p<0.001$. Similarly, VCAM-1 fell from 1,479 $(751-2,428)$ at baseline to $823(516-1,674) \mathrm{ng} / \mathrm{mL}, p<0.001$, and vWF reduced from $3,114(1,549-5,197)$ to $2,007(1,503-$ $3,542) \mathrm{mlU} / \mathrm{mL}, p=0.002$. Serum levels of hyaluronan remained unchanged. Conclusion: The combination of reduced PBR and syndecan-1 following transplant suggests that transplantation may improve glycocalyx stability at 3 months after transplant.

(C) 2021 The Author(s)

Published by S. Karger AG, Basel

\section{Introduction}

Cardiovascular disease remains the primary cause of morbidity and mortality in CKD. Endothelial dysfunction is an important factor in the development of atherosclerosis, and its presence in CKD has been demonstrated by increased flow-mediated dilation, increased intimamedia thickness, and high circulating serum markers such as vascular cell adhesion molecule-1 (VCAM-1) and von Willebrand factor (vWF) [1-3]. There are various causes of endothelial dysfunction, but a factor unique to CKD is uraemia - a state of increased inflammation and oxidative stress due to the accumulation of measured and 
unmeasured substances not excreted by failing kidneys. Vascular endothelial function becomes abnormal when exposed to uraemic toxins such as p-cresyl sulphate (PCS) and indoxyl sulphate (IS), through induction of oxidative stress [4]. Conversely, when uraemia is corrected (with haemodialysis or kidney transplantation), several studies have shown the markers of endothelial dysfunction improve significantly [5-7], indicating a strong reversible component.

The endothelium is protected by a layer, composed of proteoglycans and glycoproteins, called the glycocalyx. It has important vasculoprotective functions including mediating shear stress, governing vessel permeability to fluid and albumin, and mitigating leukocyte-endothelial interactions [8]. Assessment of this layer is commonly performed by detecting its shed components such as hyaluronan and syndecan-1 in peripheral blood. The development of novel technologies such as GlycoCheck ${ }^{\mathrm{TM}}$ has also enabled indirect assessment in vivo by sublingual imaging of the microcirculation using a handheld camera. This tool has been used in different clinical diseases such as diabetes, sepsis, heart failure, and kidney disease [9-12].

Clinically, damage to the glycocalyx has been demonstrated in various pathophysiological states including kidney disease $[13,14]$. We have previously shown an association between uraemia and damage to the glycocalyx in CKD [12]. However, it is not known whether amelioration of the uraemic environment by kidney transplantation results in measurable changes in the glycocalyx over time. We hypothesized that the reduction of uraemia after transplantation results in an improved structural stability of the glycocalyx and aimed to investigate the impact of transplantation with reduction in uraemia on the glycocalyx.

\section{Methods}

\section{Patients}

We prospectively recruited patients with end-stage kidney disease who were active on the deceased donor kidney transplant waiting list or were planned for live donor kidney transplant. We performed baseline investigations prior to the transplant procedure and repeated them 1 and 3 months after transplantation. We also recruited healthy participants with no kidney disease as controls.

\section{Investigations}

Blood was taken for full blood count, urea and electrolytes, and liver function tests. Additional serum and plasma samples were stored at $-80^{\circ} \mathrm{C}$ for subsequent analyses by enzyme-linked immunosorbent assays for markers of the glycocalyx such as hyaluronan
(R\&D Systems, Minneapolis, MN, USA) and syndecan-1 (Diaclone, Besançon, France), endothelial dysfunction such as vWF (Assaypro, Massachussets) and VCAM-1 (R\&D Systems, Minneapolis, MN, USA), and markers of uraemic toxins including PCS and IS by high-performance liquid chromatography.

Glycocalyx imaging was performed using GlycoCheck ${ }^{\mathrm{TM}}$ (Maastricht, Netherlands), consisting of a handheld Capiscope camera placed sublingually for automated video capture of the vasculature. The images were then analyzed by the GlycoCheck ${ }^{\mathrm{TM}}$ software according to the algorithm described by Lee et al. [15]. In brief, this identifies all available microvessels between 5 and $25 \mu \mathrm{m}$ in the field of view and detects erythrocytes in blood vessels through light-emitting diodes. By measuring the width of erythrocyte columns and the distance from the erythrocyte-impermeable glycocalyx margin, an averaged perfused boundary region (PBR) reading is calculated [8]. All readings were performed by a single operator (H.L.) to reduce bias.

\section{Statistics}

Based on a previous study [16], 10 participants were required in a longitudinal study to detect a $0.2-\mu \mathrm{m}$ change of the glycocalyx with $80 \%$ power and a 2 -sided alpha of 0.05 , whereas 17 patients were required per group to assess a disease state compared to controls. Comparison between 2 groups was performed using independent $t$ tests for normally distributed data or Mann-Whitney for non-parametric data. Paired $t$ tests and Kruskal-Wallis were performed before and after transplantation for parametric and nonparametric data, respectively.

\section{Results}

We recruited 30 healthy controls and 20 patients with end-stage kidney disease from April 2016 to February 2018 from a single centre. Seventeen patients were transplanted, 9 of whom received a cadaveric kidney and 8 who underwent a live donor kidney transplant. Five patients were transplanted prior to requiring dialysis. Of those who were on dialysis, the median time on dialysis was 33 (range 14-67) months. The median time from baseline assessment to transplant was a median of 93 (range 2-589) days. Patient demographics and clinical characteristics of the transplanted patients compared to healthy controls are outlined in Table 1.

Before transplantation, serum markers of glycocalyx and endothelial dysfunction at baseline were higher in patients with kidney disease compared to controls. No difference was detected in the width of the PBR between potential kidney transplant recipients and controls $(2.03$ [1.52-2.81] vs. $2.22[1.29-2.73] \mu \mathrm{m}, p=0.34$ ).

At 1 month after transplant, no patients required dialysis. Four patients had features of early humoral and cellular rejection. There was a significant reduction in haemoglobin, eGFR, IS, and PCS concentrations at 1 
Table 1. Demographics and clinical characteristics of healthy controls and transplanted patients

\begin{tabular}{|c|c|c|c|c|}
\hline & \multirow{2}{*}{$\begin{array}{l}\text { Controls } \\
(n=30)\end{array}$} & \multicolumn{3}{|c|}{ Transplants $(n=17)$} \\
\hline & & $\begin{array}{l}\text { baseline before } \\
\text { transplant }\end{array}$ & $\begin{array}{l}1 \text { month after } \\
\text { transplant }\end{array}$ & $\begin{array}{l}3 \text { months after } \\
\text { transplant }\end{array}$ \\
\hline Male/female & $11 / 19$ & $14 / 3$ & - & - \\
\hline Age, years & $36(22-69)$ & $51(25-69)$ & - & - \\
\hline \multicolumn{5}{|l|}{ Smoking status, \% } \\
\hline Never & 83 & 35 & - & - \\
\hline Ex-smoker & 17 & 65 & & \\
\hline Diabetes, $n(\%)$ & 0 & $6(35)$ & - & - \\
\hline Hypertension, $n(\%)$ & 0 & $13(76)$ & - & - \\
\hline \multicolumn{5}{|l|}{ Cause of CKD, $n(\%)$} \\
\hline IgA nephropathy & - & $6(35)$ & - & - \\
\hline Diabetes & - & $5(29)$ & - & - \\
\hline Reflux nephropathy & - & $2(12)$ & - & - \\
\hline Hypertension & - & $2(12)$ & - & - \\
\hline Polycystic kidneys & - & $1(6)$ & - & - \\
\hline FSGS & - & $1(6)$ & - & - \\
\hline $\mathrm{Hb}, \mathrm{g} / \mathrm{L}$ & $141 \pm 12$ & $115 \pm 10^{\ddagger}$ & $100 \pm 16^{\Sigma}$ & $121 \pm 12$ \\
\hline eGFR, $\mu \mathrm{mol} / \mathrm{L}$ & $98(70-127)$ & $7(4-21)^{\ddagger}$ & $57(16-65)^{\Sigma}$ & $52(21-88)^{*}$ \\
\hline ALT, IU/L & $21 \pm 11$ & $18 \pm 9$ & $17 \pm 7$ & $19 \pm 10$ \\
\hline $\mathrm{IS}, \mu \mathrm{M}$ & $1.2 \pm 0.40$ & $14.3 \pm 10.7^{\ddagger}$ & $1.6 \pm 1.2^{\Sigma}$ & $1.5 \pm 1.4^{*}$ \\
\hline $\mathrm{pCS}, \mu \mathrm{M}$ & $5(0.1-21)$ & $54(0.6-117)^{\ddagger}$ & $9(0.1-25)^{\Sigma}$ & $8(0.1-23)^{*}$ \\
\hline
\end{tabular}

Biochemical parameters of the patients at different post-transplant periods. IS, indoxyl sulphate; pCS, p-cresyl sulphate. ${ }^{\ddagger} p<0.05$ controls versus baseline. ${ }^{\Sigma} p<0.05$ baseline versus 1 month after transplant. ${ }^{*} p<0.05$ baseline versus 3 months after transplant.

month, but no glycocalyx or endothelial dysfunction changes were evident.

Three months after transplantation, markers of the glycocalyx as measured by PBR and syndecan-1 were improved. PBR decreased from to $2.22(1.29-2.73)$ to 1.98 (1.65-2.25) $\mu \mathrm{m}, p=0.024$ (Fig. 1), and syndecan- $1 \mathrm{im}-$ proved from $98(40-529)$ to $36(20-328) \mathrm{ng} / \mathrm{mL}, p<0.05$, a level similar to healthy controls $(30[12-138] \mathrm{ng} / \mathrm{mL}$, $p=0.42$ ). Similarly, markers of endothelial dysfunction improved from baseline to end of the study, albeit still significantly higher at 3 months compared to healthy controls (Fig. 2). However, serum hyaluronan levels remained unchanged throughout (Table 2).

By the end of 3 months, 2 patients still had evidence of humoral rejection. When patients with rejection were excluded from analysis $(n=4), \mathrm{PBR}$, syndecan- $1, \mathrm{vWF}$, and VCAM-1 were still significantly different from baseline compared to the study end. There were no differences in the PBR, syndecan-1, hyaluronan, or vWF at any time point between those with rejection and those without. However, in those with rejection, VCAM-1 was significantly higher compared to those without rejection at 1

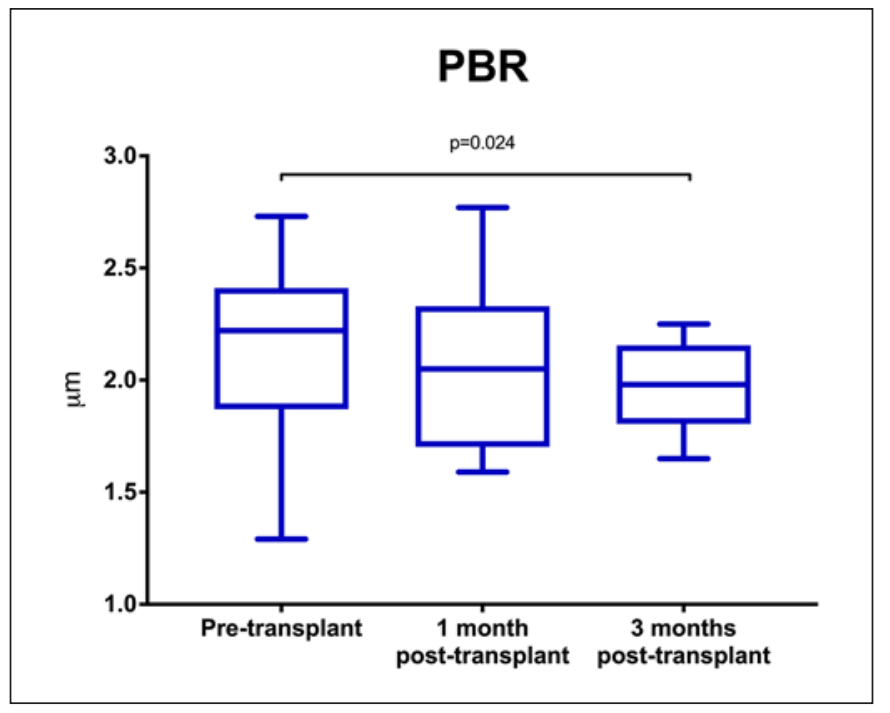

Fig. 1. Box plot of PBR values (median and range) in patients before and after transplantation. CKD patients recruited before transplant had sublingual imaging to assess glycocalyx width by the PBR. At 1 month after transplant, PBR was not different, but changes were seen after 3 months. Bars represent median and range. $\mathrm{PBR}$, perfused boundary region. 


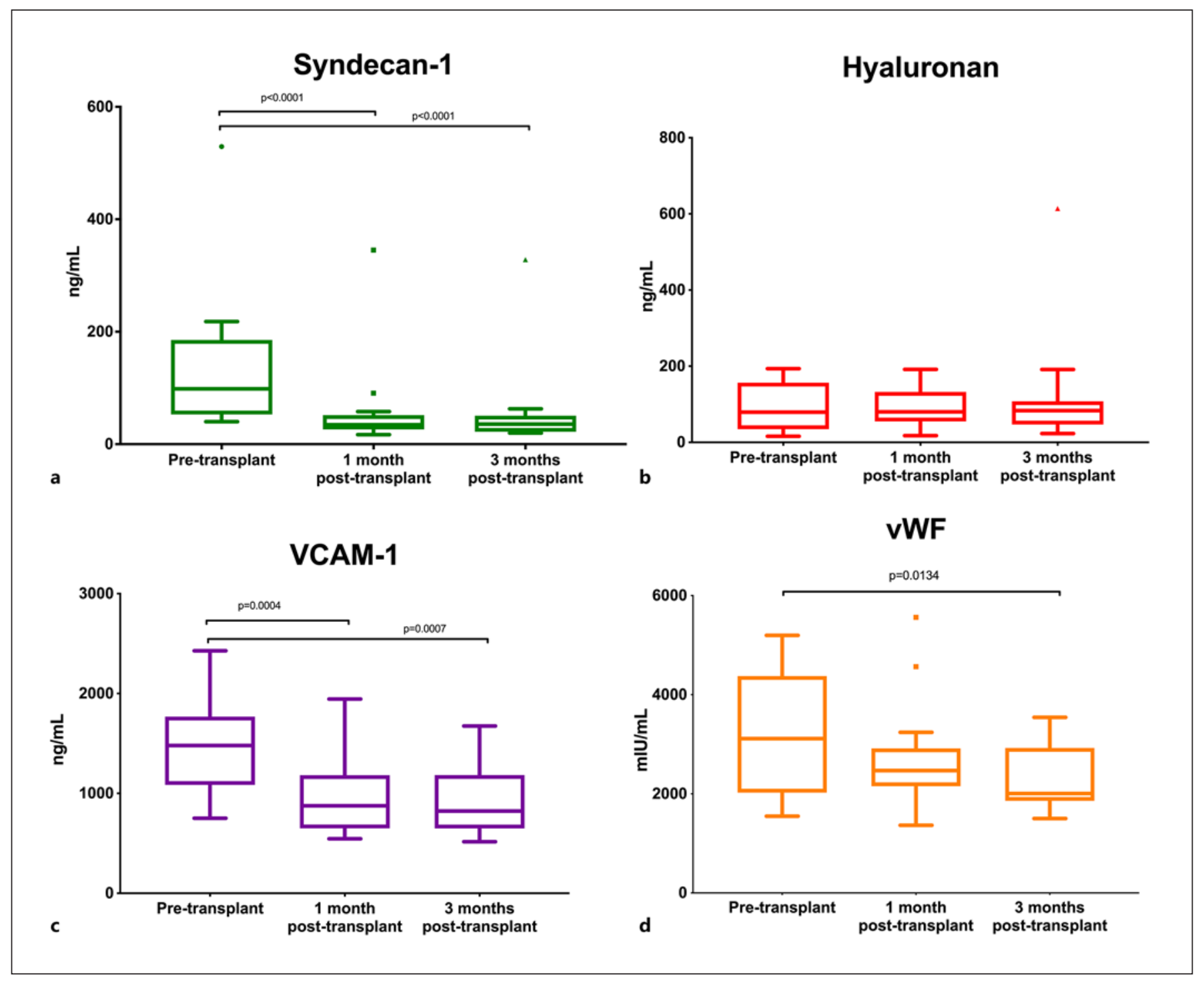

Fig. 2. Serum markers of glycocalyx damage and endothelial dysfunction before and after transplant. Syndecan-1 (a) and VCAM-1 (c) levels were improved 1 month after transplant and sustained at 3 months. vWF (d) concentrations were reduced after 3 months. Hyaluronan (b) remained unchanged. VCAM-1, vascular cell adhesion molecule; vWF, von Willebrand factor.

Table 2. Markers of glycocalyx damage and endothelial dysfunction of the patients at different post-transplant periods

\begin{tabular}{lcccc}
\hline & $\begin{array}{l}\text { Controls } \\
(n=30)\end{array}$ & \multicolumn{2}{l}{ Transplants $(n=17)$} & \\
\cline { 2 - 5 } & & baseline before transplant & 1 month after transplant & 3 months after transplant \\
\hline PBR, $\mu \mathrm{m}$ & $2.03(1.52-2.81)$ & $2.22(1.29-2.73)$ & $2.05(1.59-2.77)$ & $1.98(1.65-2.25)^{*}$ \\
Hyaluronan, ng/mL & $23(8-116)$ & $79(16-193)^{\ddagger}$ & $81(17-3,570)$ & $83(23-614)^{\psi}$ \\
Syndecan-1, ng/mL & $30(12-138)$ & $98(40-529)^{\ddagger}$ & $35(17-345)$ & $36(20-328)^{*}$ \\
vWF, $\mathrm{mIU} / \mathrm{mL}$ & $1,539(142-3,247)$ & $3,114(1,549-5,197)^{\ddagger}$ & $2,469(1,365-5,560)$ & $2,007(1,503-3,542)^{*} \psi$ \\
VCAM-1, ng/mL & $598(345-1,189)$ & $1,479(751-2,428)^{\ddagger}$ & $877(546-1,945)$ & $823(516-1,674)^{*} \psi$ \\
\hline
\end{tabular}

PBR, perfused boundary region; VCAM-1, vascular cell adhesion molecule; vWF, von Willebrand factor. ${ }^{*} p<0.05$ baseline versus 3 months after. ${ }^{\ddagger} p<0.05$ baseline versus controls. ${ }^{\Psi} p<0.05$ controls versus 3 months after. 
month $(1,795[837-1,945]$ vs. $757[545-1,228] \mathrm{ng} / \mathrm{mL}$, $p=0.03)$ and 3 months $(1,329[1,103-1,518]$ vs. $712[516-$ $1,674] \mathrm{ng} / \mathrm{mL}, p=0.015)$.

In this study, we did not find a correlation between PBR and syndecan-1 $(r=-0.08, p=0.6)$ or hyaluronan $(r=-0.13, p=0.36)$. PBR did not correlate with markers of endothelial dysfunction or uraemic toxins. However, syndecan-1 correlated with vWF $(r=0.34, p=0.02)$, VCAM-1 $(r=0.45, p=0.0014)$, IS $(r=0.41, p=0.003)$, and PCS $(r=0.45, p=0.0009)$. PBR showed a near-significant negative correlation of $r=-0.28, p=0.052$, with haemoglobin.

\section{Discussion}

To the best of our knowledge, this is the first study to assess glycocalyx changes in kidney disease before and after improvement in kidney function by kidney transplant, with the novel finding of PBR width reduction following transplantation. A previous study investigated the impact of kidney and simultaneous pancreas-kidney transplantation on endothelial dysfunction and changes in the microvasculature by sublingual imaging, but did not assess the glycocalyx [17]. Another study examined serum glycocalyx markers performed $30 \mathrm{~min}$ after surgery but did not perform sublingual imaging studies [18]. In our study, both imaging and serum markers were utilized to assess the glycocalyx before and after transplantation, allowing us to correlate both techniques. Another study in lung transplantation assessed the glycocalyx by serum markers up to 4 days after transplant [19]. However, the immediate post-transplant period can be fraught with complications associated with recent surgery and hospitalization, which may confound assessment of the glycocalyx. For example, anaemia is common after surgery and may affect PBR measurements, as demonstrated by the near-significant correlation between PBR and haemoglobin. Patients in this cohort experienced a mild reduction in the haemoglobin after surgery but recovered to pre-surgery levels by the end of the study. Despite this, the PBR demonstrated an improvement at 3 months, indicating improvement of the glycocalyx independent of the haemoglobin. Furthermore, no PBR difference was seen before transplantation between potential kidney transplant recipients (who had lower haemoglobin levels) compared to healthy controls ( $115 \pm 10$ vs. $141 \pm 12 \mathrm{~g} / \mathrm{dL}$, $p<0.001$ ).

Currently, the day-to-day variation of the glycocalyx is unknown, and there are very few studies using the PBR to determine glycocalyx changes over time. Mouse models indicate a rapid response of the glycocalyx to sepsis with near-complete destruction within 30 min but may take up to 7 days to recover in vitro $[20,21]$. By performing follow-up assessments at 1 and 3 months following kidney transplant surgery, patients in our study were provided time to stabilize clinically.

Before transplantation, the higher serum markers of glycocalyx damage and endothelial dysfunction in patients with kidney disease compared to healthy controls likely reflect their comorbid diabetes, hypertension, and renal impairment (Table 1) as these are known to have detrimental effects on the glycocalyx [22-24]. One month after transplantation, no PBR changes were evident compared to baseline, but there was a significant improvement after 3 months after transplant. The reason for this is unclear but may relate to delayed recognition of the glycocalyx reconstitution by sublingual imaging. While the sublingual microcirculation is the easiest mucosal surface to access in the human body, it may not necessarily correlate with renal microcirculation [25]. Other studies have also demonstrated a more pronounced improvement in the endothelium 12 months after transplantation, although some changes were evident after 1 month $[17,26]$.

In contrast, syndecan-1 appears to be a more sensitive marker of glycocalyx change, detecting improvement at 1 month (and sustained after 3 months) and correlating with uraemic toxins levels. Syndecan- 1 consists of various domains which traverse the endothelial cell membrane, shed by matrix metalloproteinases (MMP) in response to inflammation and injury. Uraemic toxins activate epidermal growth factor receptors, which in turn induce MMP. While the activity of MMP in the immediate transplant period is unclear, it is conceivable that reduced levels of uraemic toxins after transplantation similarly reduce the induction of MMP levels.

Hyaluronan, in contrast, is neither bound to a core protein nor anchored to the endothelial cell membrane. This may cause hyaluronan to be more susceptible to shear forces and shedding [27], which may account for the persistently elevated levels observed at 3 months. In other studies, hyaluronan decreased to normal values following successful renal transplantation; however, levels were unaltered when measured on 2 occasions 4-15 months apart [28]. Furthermore, as this study was powered to detect a PBR change, a larger sample size may be required to assess changes in hyaluronan levels.

The influence of renal clearance on glycocalyx markers is debatable. Hyaluronan is a large molecule of up to 
$4,000 \mathrm{kDa}$ which is primarily cleared by the liver. It is degraded into smaller fragments, some of which are handled by the renal circulation. Circulating hyaluronan in CKD consists primarily of high molecular weight hyaluronan, and therefore renal clearance is unlikely to contribute to its metabolism [29]. Furthermore, improvement in the kidney function of these patients did not alter hyaluronan levels, and liver function as measured by ALT was unchanged. However, information on syndecan-1 handling is limited. Syndecan-1 is removed from the cell surface by endocytosis and lysosomal degradation, as well as cleavage by MMPs, chemokines, and bacterial virulence factors [30, 31]. However, the contribution of renal clearance on the metabolism of syndecan-1 is unknown. In this study, the change in eGFR correlates with the change in syndecan-1 levels ( $\delta$ eGFR and $\delta$ syndecan, $r=-0.41, p=0.0034$ ), suggesting syndecan-1 may be cleared by the kidneys as renal function improves.

The impact of acute rejection on the glycocalyx has not yet been defined. The endothelium has been implicated in the development of chronic transplant rejection $[32,33]$, and kidney transplant recipients with interstitial fibrosis and tubular atrophy had a higher PBR compared to healthy controls or those with normal functioning kidney transplants [14]. In this study, patients with acute rejection did not have worse glycocalyx parameters, likely due to the short follow-up period and small number of participants. Despite this, elevated VCAM-1 levels were detectable early in the course of rejection. This is not surprising, as VCAM-1 is widely expressed in the peritubular capillaries during transplant rejection, associated with leukocyte infiltration of these vessels [34].

There are several limitations to this study. The possible impact of medications on the glycocalyx must be considered, particularly immunosuppressants. Mycophenolic acid has endothelial protective properties, whereas calcineurin inhibitors can damage the endothelium [35]. Glucocorticoids, a potent anti-inflammatory agent, have demonstrated positive effects on the endothelium [36]. There was also a wide variation in the time between baseline assessment and transplant as we recruited patients awaiting deceased donor transplantation where time-totransplant was unpredictable. Healthy controls were not re-examined after the initial assessment, but as the glycocalyx is thought to be in a steady state of synthesis and degradation in health, their glycocalyx markers are not expected to be different [37].

\section{Conclusion}

In this single-centre, prospective study of patients with kidney disease, renal transplantation results in improved markers of the glycocalyx, endothelial dysfunction, and uraemic toxins up to 3 months after transplant. A novel finding of this study is the reduction in the width of the PBR following transplant. Whether this is a direct effect of improved kidney function or other factors remains to be determined.

\section{Statement of Ethics}

This study was approved by the Ethics Committee of Eastern Health (HREC/15/EH/272, Melbourne, Australia), and written informed consent was obtained from all subjects.

\section{Conflict of Interest Statement}

The authors have no conflicts of interest to declare.

\section{Funding Sources}

This work was supported by Monash University in Melbourne, Australia.

\section{Author Contributions}

H.L. was involved in planning the research, literature review, collecting data, interpretation of results, statistical analysis, and writing the manuscript. M.R. was involved in interpretation of results, statistical analysis, and writing the manuscript. L.P.M. was involved in coordination and supervision of research, interpretation of results, and writing the manuscript.

\section{References}

1 Vaccaro F, Mulè G, Cottone S, Soresi M, Giannitrapani L, Vadalà A, et al. Circulating levels of adhesion molecules in chronic kidney disease correlate with the stage of renal disease and with C-reactive protein. Arch Med Res. 2007;38(5):534-8.

2 Yilmaz MI, Stenvinkel P, Sonmez A, Saglam M, Yaman H, Kilic S, et al. Vascular health, systemic inflammation and progressive reduction in kidney function; clinical determinants and impact on cardiovascular outcomes. Nephrol Dial Transplant. 2011;26(11):3537-43.

3 Thambyrajah J, Landray MJ, McGlynn FJ, Jones HJ, Wheeler DC, Townend JN. Abnormalities of endothelial function in patients with predialysis renal failure. Heart. 2000; 83(2):205-9. 
4 Harper SJ, Tomson CR, Bates DO. Human uremic plasma increases microvascular permeability to water and proteins in vivo. Kidney Int. 2002;61(4):1416-22.

5 Yilmaz MI, Saglam M, Carrero JJ, Qureshi AR, Caglar K, Eyileten T, et al. Normalization of endothelial dysfunction following renal transplantation is accompanied by a reduction of circulating visfatin/NAMPT. A novel marker of endothelial damage? Clin Transplant. 2009;23(2):241-8.

6 Cross JM, Donald A, Vallance PJ, Deanfield JE, Woolfson RG, MacAllister RJ. Dialysis improves endothelial function in humans. Nephrol Dial Transplant. 2001;16(9):1823-9.

7 Oflaz H, Turkmen A, Turgut F, Pamukcu B, Umman S, Ucar A, et al. Changes in endothelial function before and after renal transplantation. Transpl Int. 2006;19(4):333-7.

8 Liew H, Roberts MA, MacGinley R, McMahon LP. Endothelial glycocalyx in health and kidney disease: rising star or false dawn? Nephrology. 2017;22(12):940-6.

9 Martens RJ, Vink H, van Oostenbrugge RJ, Staals J. Sublingual microvascular glycocalyx dimensions in lacunar stroke patients. Cerebrovasc Dis. 2013;35(5):451-4.

10 Donati A, Damiani E, Domizi R, Romano R, Adrario E, Pelaia P, et al. Alteration of the sublingual microvascular glycocalyx in critically ill patients. Microvasc Res. 2013;90:869.

11 Wadowski PP, Hülsmann M, Schörgenhofer C, Lang IM, Wurm R, Gremmel T, et al. Sublingual functional capillary rarefaction in chronic heart failure. Eur J Clin Invest. 2018; $48(2)$.

12 Liew H, Roberts MA, Pope A, McMahon LP. Endothelial glycocalyx damage in kidney disease correlates with uraemic toxins and endothelial dysfunction. BMC Nephrol. 2021; 22(1):21.

13 Vlahu CA, Lemkes BA, Struijk DG, Koopman MG, Krediet RT, Vink H. Damage of the endothelial glycocalyx in dialysis patients. J Am Soc Nephrol. 2012;23(11):1900-8.

14 Dane MJ, Khairoun M, Lee DH, van den Berg BM, Eskens BJ, Boels MG, et al. Association of kidney function with changes in the endothelial surface layer. Clin J Am Soc Nephrol. 2014;9(4):698-704.

15 Lee DH, Dane MJ, van den Berg BM, Boels MG, van Teeffelen JW, de Mutsert R, et al. Deeper penetration of erythrocytes into the endothelial glycocalyx is associated with impaired microvascular perfusion. PLoS One. 2014;9(5):e96477.

16 Nieuwdorp M, Meuwese MC, Mooij HL, Ince C, Broekhuizen LN, Kastelein JJ, et al. Measuring endothelial glycocalyx dimensions in humans: a potential novel tool to monitor vascular vulnerability. J Appl Physiol. 2008; 104(3):845-52.

17 Khairoun M, de Koning EJ, van den Berg BM, Lievers E, de Boer HC, Schaapherder AF, et al. Microvascular damage in type 1 diabetic patients is reversed in the first year after simultaneous pancreas-kidney transplantation. Am J Transplant. 2013;13(5):1272-81.

18 Snoeijs MG, Vink H, Voesten N, Christiaans MH, Daemen JW, Peppelenbosch AG, et al. Acute ischemic injury to the renal microvasculature in human kidney transplantation. Am J Physiol Renal Physiol. 2010;299(5): F1134-40.

19 Sladden TM, Yerkovich S, Grant M, Zhang F, Liu X, Trotter M, et al. Endothelial glycocalyx shedding predicts donor organ acceptability and is associated with primary graft dysfunction in lung transplant recipients. Transplantation. 2019;103(6):1277-85.

20 Yang Y, Yang G, Schmidt EP. In vivo measurement of the mouse pulmonary endothelial surface layer. J Vis Exp. 2013;(72):e50322.

21 Potter DR, Jiang J, Damiano ER. The recovery time course of the endothelial cell glycocalyx in vivo and its implications in vitro. Circ Res. 2009; 104(11):1318-25.

22 Dogne S, Flamion B, Caron N. Endothelial glycocalyx as a shield against diabetic vascular complications: involvement of hyaluronan and hyaluronidases. Arterioscler Thromb Vasc Biol. 2018;38(7):1427-39.

23 Ikonomidis I, Voumvourakis A, Makavos G, Triantafyllidi H, Pavlidis G, Katogiannis K, et al. Association of impaired endothelial glycocalyx with arterial stiffness, coronary microcirculatory dysfunction, and abnormal myocardial deformation in untreated hypertensives. J Clin Hypertens. 2018;20(4): 672-9.

24 Padberg JS, Wiesinger A, di Marco GS, Reuter S, Grabner A, Kentrup D, et al. Damage of the endothelial glycocalyx in chronic kidney disease. Atherosclerosis. 2014;234(2):335-43.

25 Sui F, Zheng Y, Li WX, Zhou JL. Renal circulation and microcirculation during intra-abdominal hypertension in a porcine model.
Eur Rev Med Pharmacol Sci. 2016;20(3):45261.

26 Bijkerk R, Duijs JM, Khairoun M, Ter Horst CJ, van der Pol P, Mallat MJ, et al. Circulating microRNAs associate with diabetic nephropathy and systemic microvascular damage and normalize after simultaneous pancreas-kidney transplantation. Am J Transplant. 2015; 15(4):1081-90.

27 Reitsma S, Slaaf DW, Vink H, van Zandvoort MA, oude Egbrink MG. The endothelial glycocalyx: composition, functions, and visualization. Pflugers Arch. 2007;454(3):345-59.

28 Turney JH, Davison AM, Forbes MA, Cooper $\mathrm{EH}$. Hyaluronic acid in end-stage renal failure treated by haemodialysis: clinical correlates and implications. Nephrol Dial Transplant. 1991;6(8):566-70.

29 Woodrow G, Turney JH, Davison AM, Cooper EH. Serum hyaluronan concentrations predict survival in patients with chronic renal failure on maintenance haemodialysis. Nephrol Dial Transplant. 1996;11(1):98-100.

30 Carey DJ. Syndecans: multifunctional cellsurface co-receptors. Biochem J. 1997;327(Pt 1): $1-16$.

31 Hayashida K, Stahl PD, Park PW. Syndecan-1 ectodomain shedding is regulated by the small GTPase Rab5. J Biol Chem. 2008; 283(51):35435-44.

32 Denton MD, Davis SF, Baum MA, Melter M, Reinders ME, Exeni A, et al. The role of the graft endothelium in transplant rejection: evidence that endothelial activation may serve as a clinical marker for the development of chronic rejection. Pediatr Transplant. 2000; 4(4):252-60.

33 Al-Lamki RS, Bradley JR, Pober JS. Endothelial cells in allograft rejection. Transplantation. 2008;86(10):1340-8.

34 Hill PA, Main IW, Atkins RC. ICAM-1 and VCAM-1 in human renal allograft rejection. Kidney Int. 1995;47(5):1383-91.

35 Cardinal H, Dieudé M, Hébert MJ. Endothelial dysfunction in kidney transplantation. Front Immunol. 2018;9:1130.

36 Gokalp O, Iscan S, Iner H, Gurbuz A. The effects of steroids on endothelial function. Anatol J Cardiol. 2017;18(4):309.

37 Kolarova H, Ambruzova B, Svihalkova Sindlerova L, Klinke A, Kubala L. Modulation of endothelial glycocalyx structure under inflammatory conditions. Mediators Inflamm. 2014;2014:694312. 